\title{
-NOTES-
}

\section{AN INEQUALITY FOR THE AMPLITUDES AND AREAS IN VIBRATION DIAGRAMS OF TIME-DEPENDENT FREQUENCY*}

By PHILIP HARTMAN AND AUREL WINTNER (The Johns Hopkins University)

Let $\omega(t)$ be any real-valued, continuous function on a $t$ interval, and let $x(t)$ be any real-valued, non-trivial $(\not \equiv 0)$ solution of

$$
x^{\prime \prime}+\omega^{2}(t) x=0 .
$$

Unless $\omega(t)$ is of some extremely particular type, no solution $x(t)$ of this general form, (1), of the problem of frequency modulation can be found "explicitly" (the actual case of frequency modulation is the particular case

$$
\omega^{2}(t)=1 /(\alpha+\beta \cos t), \quad(|\beta|<\alpha) ;
$$

but (1) cannot be integrated in explicit terms in this case either).

It is therefore of interest that, without solving (1), one can delimit an explicit inequality for the maximum amplitude and the area of every half-wave (if any) of an arbitrary solution of every differential equation of the form (1). This is the content of (4) below. It will also be shown that the inequality in question is the best possible of its kind (if $\omega(t)$ is unspecified).

Unless the given $t$-interval is either too short or such that $\omega(t)$ is "too close to 0 " on it (i.e., (1) is "too close to $x^{\prime \prime}=0$," with $x(t)=c_{1} t+c_{2}$ ), a solution $x(t)$ will have more than one zero, say $x(a)=0$ and $x(b)=0$, where $a<b$. Then, since $x(t)$ is not the trivial solution, it can be assumed that $t=b$ is the first zero following the zero $t=a$; so that $x(t) \neq 0$ if $a<t<b$. Since $-x(t)$ is a solution of $(1)$ if $x(t)$ is, there is no further loss of generality in assuming that

$$
x(t)>0 \text { if } a<t<b \text {, while } x(a)=0, x(b)=0 .
$$

Let $M$ denote the maximum amplitude, and $A$ the area, of such a half-wave in the $(t, x)$-plane, that is, put

$$
M_{\mathrm{I}}=\max _{a<t<b} x(t), \quad A=\int_{a}^{b} x(t) d t .
$$

It will be shown that, no matter what the frequency function $\omega(t)$ of (1) may be, the geometrical characteristics (3) of every half-wave (2) of every solution $x(t)$ are subject to the inequality

$$
M / A<\frac{1}{2} \int_{a}^{b} \omega^{2}(t) d t .
$$

It will also be shown that the $\frac{1}{2}$ in (4) is the best absolute constant, in the sense that the $\frac{1}{2}$ cannot be replaced by any numerical constant less than $\frac{1}{2}$, valid for every differential equation (1). 
First, it is clear from (1) that $x^{\prime \prime}(t)$ is non-positive if and only if $x(t)$ is non-negative. It follows therefore from $(2)$ that the $(t, x)$-graph represents a convex arch over the segment $[a, b]$ of the $t$-axis. Needless to say, the line $t=c$, where $c$ denotes the abscissa belonging to the maximum (for $a \leq t \leq b$ ) of the ordinate, need not be a line of $x y m-$ metry of the arch. But it is clear from the convexity of the latter that the triangle having the base $[a, b]$ and the vertex $t=c, x=x(c)$ is situated beneath the arch. In particular, this triangle has a smaller area than the convex region surrounded by the segment $[a, b]$ and the arch. In view of $(3)$, this means that

$$
A>\frac{1}{2}(b-a) M \text {. }
$$

On the other hand, by a well-known inequality which goes back to Liapounoff, ${ }^{1}$

$$
\int_{a}^{b} \omega^{2}(t) d t>4 /(b-a) \text {. }
$$

Clearly, (6) and (5) together imply (4).

It is also clear that the inequality (5) turns into an inequality when the convex arch degenerates into two sides of the triangle considered above. On the other hand, the 4 occurring in (6) is the best possible absolute constant, ${ }^{2}$ and the optimal nature of this 4 can also be concluded by confining the convex arch to be arbitrarily close to the triangular form. ${ }^{3}$ Accordingly, the best absolute constants, $\frac{1}{2}$ and 4 , occurring in (5) and (6) respectively, can be approximated by the same sequence of examples. This proves the statement italicized after (4).

${ }^{1}$ See G. Borg, Amer. Jour. Math. 71, 67-70 (1949). For a proof embedding (6) into a general theory, cf. A. Wintner, ibid. 73, 368-380 (1951). For refinements of (6), cf. P. Hartman and A. Wintner, ibid. 73 885-890 (1951).

${ }^{2}$ E. R. van Kampen and A. Wintner, ibid, 59, 270-274 (1937).

${ }^{3} \mathrm{G}$. Borg, loc. cit.

\section{A FORMULA FOR THE NORMALIZATION CONSTANT IN EIGEN VALUE PROBLEMS*}

By GOTTFRIED GUDERLEY

Given the differential equation

$$
y^{\prime \prime}+\lambda r(x) y=0
$$

with suitable homogeneous boundary conditions at the end of an interval which extends from $x=a$ to $x=b$. In (1) $\lambda$ denotes an eigen value. During the following derivation $\lambda$ is considered as arbitrary and only the boundary condition at one end of the interval, say point $a$, will be imposed, so the functions $y$ need not be eigen functions. If $y=y_{\mathrm{I}}$ 